\title{
(C) OPEN ACCESS \\ Modelling the impact of a new tobacco product: review of Philip Morris International's Population Health Impact Model as applied to the IQOS heated tobacco product
}

\author{
Wendy B Max, ${ }^{1,2}$ Hai-Yen Sung, ${ }^{1,2}$ James Lightwood, ${ }^{3}$ Yingning Wang, ${ }^{1,2}$ Tingting Yao ${ }^{1,2}$
}

\begin{abstract}
- Additional material is published online only. To view please visit the journal online (http://dx.doi.org/10.1136/ tobaccocontrol-2018-054572)

${ }^{1}$ Institute for Health and Aging, School of Nursing, University of California, San Francisco, California, USA

${ }^{2}$ Department of Social and Behavioral Sciences, School of Nursing, San Francisco, California, USA

${ }^{3}$ Department of Clinical Pharmacy, School of Pharmacy, San Francisco, California, USA
\end{abstract}

\section{Correspondence to}

Professor Wendy B Max, Institute for Health and Aging, University of California, San Francisco, CA 94118, USA; wendy.max@ucsf.edu

Received 15 June 2018 Revised 6 September 2018 Accepted 7 September 2018 Published Online First 1 October 2018

\section{Check for updates}

(C) Author(s) (or their employer(s)) 2018. Re-use permitted under CC BY-NC. No commercial re-use. See rights and permissions. Published by BMJ.

To cite: Max WB, Sung $\mathrm{H}-\mathrm{Y}$, Lightwood J, et al. Tob Control

2018:27:s82-s86.

\begin{abstract}
Objectives We review the Population Health Impact Model (PHIM) developed by Philip Morris International and used in its application to the US Food and Drug Administration (FDA) to market its heated tobacco product (HTP), IQOS, as a modified-risk tobacco product (MRTP). We assess the model against FDA guidelines for MRTP applications and consider more general criteria for evaluating reduced-risk tobacco products.
\end{abstract}

Methods In assessing the PHIM against FDA guidelines, we consider two key components of the model: the assumptions implicit in the model (outcomes included, relative harm of the new product vs cigarettes, tobacco-related diseases considered, whether dual or polyuse of the new product is modelled, and what other tobacco products are included) and data used to estimate and validate model parameters (transition rates between non-smoking, cigarette-only smoking, dual use of cigarettes and MRTP, and MRTP-only use; and starting tobacco use prevalence).

Results The PHIM is a dynamic state transition model which models the impact of cigarette and MRTP use on mortality from four tobacco-attributable diseases. The PHIM excludes morbidity, underestimates mortality, excludes tobacco products other than cigarettes, does not include FDA-recommended impacts on non-users and underestimates the impact on other population groups.

Conclusion The PHIM underestimates the health impact of HTP products and cannot be used to justify an MRTP claim. An assessment of the impact of a potential MRTP on population health should include a comprehensive measure of health impacts, consideration of all groups impacted, and documented and justifiable assumptions regarding model parameters.

\section{INTRODUCTION}

Philip Morris International (PMI) submitted an application to the Food and Drug Administration (FDA) to market its heated tobacco product (HTP), IQOS, as a modified-risk tobacco product (MRTP) in the USA, arguing that because the product does not actually burn tobacco, it will have a reduced impact on health compared with cigarettes. PMI used a computational model they developed, the Population Health Impact Model (PHIM), ${ }^{1}$ to estimate the potential impact of this IQOS marketing on public health. While the application was denied by the FDA, the proliferation of purported reduced harm products suggests the need for an understanding of how to assess the impact on population health of new tobacco products.

No models specifically consider the health impact of IQOS, but several simulation models analyse the impact of two tobacco products on population health. These models evaluate the impact of a reduced-risk tobacco product on population health by comparing a factual scenario (considering cigarette use only) with a counterfactual scenario, in which the new product is introduced. None of the models consider the impact of other tobacco products. Details of the models, the assumptions they are based on and their findings are summarised in online supplement 1 and online supplement table 1. Four models compared the health effects of cigarettes with e-cigarettes (or a vaporised nicotine product), measuring health effects either as an index ${ }^{2}$ or as mortality. ${ }^{3-6}$ Two of these models reported a net positive impact on health ${ }^{346}$ while two reported net population harm. ${ }^{25}$ All four research teams assumed that e-cigarettes were safer than cigarettes by factors ranging from $5 \%$ to $30 \%$, but they differed in their assumptions about the impact of e-cigarettes on cigarette smoking initiation and cessation. Three studies analysed the impact of introducing a non-specified MRTP on cigarette smoking and mortality. Each study reported a potential reduction in mortality, ${ }^{7-9}$ though one study indicated that mortality could increase if the MRTP were $50 \%$ as risky as cigarettes and $50 \%$ of initiates were never smokers. ${ }^{8}$ One study ${ }^{10}$ evaluated the impact of promoting use of the smokeless product snus on a health index, and concluded that promoting snus as a safer product than cigarettes is not likely to result in population health benefits.

These models illustrate how different assumptions about what is included in the model as well as the data sources for estimating transition rates and tobacco use prevalence lead to varying conclusions about the net impact of a new product. These model characteristics will be reviewed for the PHIM.

PMI's multiple tobacco product model, the PHIM, was refined for its application for IQOS. This paper reviews the FDA guidelines for MRTP applications and assesses whether the PHIM as used in the IQOS MRTP application meets the criteria the FDA has developed to determine whether or not the impact of IQOS on population health justifies the introduction of the product as an MRTP. We also consider more generally the criteria for 
assessing the impact of a new tobacco product on population health.

\section{METHODS}

We evaluate the PHIM as published ${ }^{11}$ and as submitted for marketing IQOS as a MRTP ${ }^{12} 13$ against FDA guidelines for MRTP applications. ${ }^{14}$ In our evaluation, we consider two key components of the model: the assumptions implicit in the model (outcomes included, relative harm of the new product vs cigarettes, tobacco-related diseases considered, whether dual or polyuse of the new product is modelled, and what other tobacco products are included) and data used to estimate and validate model parameters (transition rates between non-smoking, cigarette-only smoking, dual use of cigarettes and MRTP, and MRTPonly use; and starting tobacco use prevalence).

The FDA issued draft guidelines for MRTP applications in March 2012. ${ }^{14}$ The guidelines specify that 'scientific studies submitted by the applicant "should contain an overall assessment of the potential effect that the marketing of the product as proposed may have on tobacco-related morbidity and mortality". (p21) ${ }^{14}$ The guidelines further recommend that the potential impact on mortality and morbidity be assessed for seven population groups and exposure patterns. $(\mathrm{p} 22)^{14}$

\section{RESULTS}

\section{The PMI PHIM}

The PHIM, developed by PMI researchers and their collaborators, is described briefly here and in more detail in online supplement 2. The PHIM is a dynamic state transition model which models the impact of cigarette and MRTP use on mortality. It follows a cohort aged 15 and older for 20 years. The PHIM consists of a prevalence component ('P-component') and an epidemiological risk component ('E-component'). ${ }^{1}$ The P-component models changes in the distribution of cigarette and/or MRTP use occurring in a hypothetical population over a defined period. The model compared a null (ie, no MRTP) scenario and an MRTP scenario. $(\mathrm{p} 88)^{1}$ For each scenario, transition probabilities for initiation, reinitiation and cessation of smoking and of product switching (including dual cigarette/MRTP use) are estimated from historical cigarette smoking prevalence data, and premarket Perception and Behavioral Assessment studies conducted by PMI. ${ }^{12}$ The E-component uses the tobacco use patterns from the P-component along with estimates of the relative risk (RR) of death for lung cancer, ischaemic heart disease (IHD), stroke and chronic obstructive pulmonary disease (COPD) to estimate mortality using published estimates of RR for smoking and assumptions about how much less risky MRTP use is compared with smoking.

Sensitivity analyses vary assumptions about initiation and reinitiation of tobacco use; transition rates between smoking, MRTP and dual use; time frames; and the RR of the MRTP versus cigarettes.

\section{Comparison of IQOS MRTP application with FDA guidelines Impact of IQOS on morbidity}

The PHIM does not include any measure of morbidity, such as incident or prevalent cases of tobacco-related illness. One way of quantifying the impact of morbidity is through healthcare costs which incorporate the severity and time course of illness, and would include hospitalisations, outpatient care, medications and other services. No estimates of healthcare costs are made in the PHIM.

\section{Impact of IQOS on mortality}

The PHIM considers mortality from four diseases caused by smoking-lung cancer, IHD, stroke and COPD.

The base case in the IQOS MRTP application assumes that compared with cigarettes, sole MRTP use is $80 \%$ less risky and dual use of MRTP and cigarettes is $40 \%$ less risky than cigarette smoking alone. The RR of death for dual use of cigarettes and IQOS is assumed to be the midpoint of the risk of cigarette smoking and the risk of IQOS use.(p19) ${ }^{12}$ To simulate the mortality impact on the US population, the model uses smoking prevalence from 1990 projected through 2010.

\section{Impact of IQOS on different types of individuals}

We next assess how the PHIM treats the seven population groups and exposure patterns recommended for consideration by the FDA.(p22) ${ }^{14}$ More detailed descriptions are contained in online supplement table 2 .

1. Tobacco users who switch from other commercially marketed tobacco products to the proposed product. The PHIM considers switching only from cigarettes. PMI acknowledges that other tobacco products are not considered in their model, arguing that there is no evidence to indicate that IQOS users will switch from other tobacco products. $(\mathrm{p} 7)^{12}$

2. Tobacco users and non-users who, after adopting the proposed product, switch to or switch back to other tobacco products that may present higher levels of individual health risk. The PHIM assumes that each month $0.1 \%$ of IQOS users will switch to cigarette smoking, but that after a year of IQOS use virtually no users will become cigarette smokers or dual users. They also assume that $10 \%$ of dual IQOS/ cigarette smokers will become sole cigarette smokers each month (p14) $)^{12}$ (online supplement table 4).

3. Tobacco users who opt to use the proposed product rather than cease tobacco use altogether. PMI indicates that this group was 'considered by a specific analysis in which current conventional cigarette smokers who would otherwise have switched to MRTP or to dual use, quit instead'.(p5, Module 7.4) ${ }^{13}$ PMI indicates that 'here, the reduction in deaths associated with MRTP introduction was estimated to be about 11 times greater in males and about 13 times greater in females than that for the basic analysis'.(p5, Module 7.4) ${ }^{13}$

4. Tobacco users who opt to use the proposed product rather than an FDA-approved tobacco cessation medication. PMI indicates that this is 'outside the present scope of the model'. (p5, Module 7.4) ${ }^{13}$

5. Non-users who initiate tobacco use with the proposed product, such as youth, never users, former users. The PHIM assumes that uptake of the IQOS HTP will be limited among youth because of the relatively high cost. It assumes that the per cent of never-smokers who will initiate tobacco use with IQOS each month ranges from $0.05 \%$ to $0.08 \%$ (after 25 years), and that the rate drops with age, with no one initiating use after age 35 (p13, Module 6.5) ${ }^{12}$ (online supplement table 3). The model assumes that reinitiation rates of former smokers with IQOS range from $0.01 \%$ for youth aged $15-$ 19 years to $0.08 \%$ for older adults (aged 75-79 years) after more than 25 years (p13, Module 6.5) ${ }^{12}$ (online supplement table 3).

6. Tobacco users who use the product in conjunction with other tobacco products. The PHIM assumes that few smokers or IQOS users will become dual users (p14, Module 6.5) (on- $^{12}$ (on line supplement table 4 ) and that fewer than $0.02 \%$ of never tobacco users and fewer than $0.04 \%$ of former smokers will 
become dual users ( $\mathrm{p} 13$, Module 6.5) ${ }^{12}$ (online supplement table 3).

7. Non-users who experience health risks from the product. Risk to non-users is not considered in the PHIM.

\section{DISCUSSION}

The PHIM is similar in structure to many of the published models reviewed which are all dynamic in nature and model state transitions in tobacco use over time, with the exception of one steady state model. ${ }^{2}$ The PHIM focuses on mortality as the outcome measure as do all but two models which included a health effects index. ${ }^{210}$ The PHIM models the population aged 15 and older, an improvement over some of the models which focus on a subgroup of the population. It follows the population for 20 years which is reasonable for MRTP application purposes, and is in line with the published models which use varying time horizons from 10 to 84 years.

However, the PHIM analysis of IQOS has some important limitations that are apparent in reviewing the model against FDA recommendations. Morbidity-related outcomes are omitted, mortality is underestimated, transition rates used in the model are based on PMI perception studies and the model uses data for the USA in 1990 as a starting point. The role of other tobacco products such as e-cigarettes and impact on non-users are not considered. Thus, the analysis of IQOS does not fully satisfy FDA guidelines for MRTP applications, and results in an overestimation of the benefit of IQOS on population health.

\section{Morbidity is ignored}

The PHIM does not include any measures of morbidity, such as tobacco-related disease incidence or tobacco-attributable healthcare costs, though this is an FDA requirement. Morbidity costs are more than half of total costs of cigarette smoking for high-income countries, ${ }^{15}$ so this omission is potentially serious.

\section{Mortality is underestimated}

The clinical results presented for US adults to justify the lower RR of mortality for IQOS versus cigarette use do not show statistically significant improvements in the biomarkers of harm that PMI assessed in actual people who used HTP (with a single exception). ${ }^{16}$ This contradicts the assertion of reduced harm, and does not justify the 70\%-90\% reductions in risk assumed in the PHIM. The RR of mortality for IQOS compared with cigarettes is a critical parameter in the model and a smaller reduction in harm should be used in the analyses.

The RRs of mortality from smoking used in the PHIM are based on multicountry studies rather than those published by the 2014 US Surgeon General and based on US cohorts. ${ }^{17}$ PMI's sensitivity analyses indicate that the proportion of smoking-attributable deaths from the four causes for men would increase $15 \%(2005-2009)$ if based on the RRs from the Surgeon General report, ${ }^{11}$ with less of a change for women. The Surgeon General estimates, which are more current and vetted through a more thorough process of independent peer review than the PHIM estimates, are more appropriate and should be used in these analyses.

The PHIM assumes that the RR of dual use of IQOS and cigarettes is the midpoint of the two RRs. However, there is some evidence that dual users of cigarettes and e-cigarettes have greater risks of negative health outcomes than sole cigarette users, ${ }^{18}$ suggesting that there could be greater risks for dual users of IQOS and cigarettes and that the PHIM model may underestimate the number of deaths attributable to dual use.
The inclusion of only four smoking-attributable diseases in the PHIM further reduces the estimates of mortality from IQOS versus cigarette use. At least 22 causes of death for adults ${ }^{19}$ and 4 causes of death for infants ${ }^{20}$ have been causally linked to cigarette smoking. PMI acknowledges that the 'overall estimates of deaths saved due to the introduction of IQOS would have to be increased about 50\% to give an estimate for all smoking-related diseases combined'. $(\mathrm{p} 41)^{12}$

Given that mortality is the main measure of population health used in the PHIM, the use of low RRs and the inclusion of only four causes of death will result in an overestimate of the benefit of IQOS introduction as an MRTP which will greatly impact the results.

\section{Assumptions about transition rates are not well justified}

The PHIM uses transition probabilities for smoking dating back to 1986 for 12 countries. PMI does report an adjustment for poor model forecast performance through 2005 but does not report the methodology or provide documentation of the model predictions against historical data. Other models use more recent data, document the methodology and report the predictive performance against historical data. For example, Warner and Mendez ${ }^{6}$ validate their model to US data through 2015, and Levy et $a l^{3}$ calibrate their model using US data through 2010. The absence of an explanation of methodology and documentation for the PHIM predictive performance is a serious weakness because poor forecasts of status quo and alternative scenarios may bias the results.

Transition probabilities for IQOS initiation, reinitiation, cessation and product switching are based on PMI perception surveys. The only empirical data available are from Italy, but these data report on ever use and are thus not comparable with the PHIM estimates. ${ }^{21}$ Youth have initiated tobacco use with e-cigarettes at high rates, ${ }^{22}$ and may find the IQOS product to be similarly appealing. Flavours, electronic features and perceptions of harm are factors that are important determinants of adolescent decisions regarding tobacco use, and IQOS is likely to appeal to them on all these characteristics. ${ }^{23}$ The PHIM assumption that youth uptake will be limited because of the relatively high cost ignores the use of coupons to reduce prices, a common tobacco industry pricing strategy, and also ignores shared use among users. Recent estimates suggest that the prevalence of sharing e-cigarette devices among adolescents over the previous 30 days exceeds $70 \%$. $^{24}$

The PHIM makes optimistic assumptions about cigarette smoking cessation rates associated with IQOS use, assuming that $0.4 \%-1.5 \%$ of smokers will quit smoking each month due to IQOS use,(Module 6.5) ${ }^{12}$ (online supplement table 3) a relatively high rate in light of evidence that many IQOS users continue to smoke cigarettes, including PMI's own finding that $36 \%$ of Japanese IQOS users use another tobacco product. ${ }^{25}$ Recent research has produced evidence for the USA that, with the current regulatory environment and smoking behaviours, e-cigarettes do not increase smoking cessation in the general population greater than what would have occurred without them. ${ }^{26}$ Furthermore, the potential effectiveness of e-cigarettes in aiding smoking cessation may depend greatly on the level of the smoker's nicotine dependence. ${ }^{27}$ This is also likely to impact the effectiveness of IQOS in cessation, but is not acknowledged in the PHIM.

The potential gateway effect of IQOS is not fully considered. There is evidence for youth and young adults that e-cigarette use increases subsequent uptake of cigarette smoking. ${ }^{28} \mathrm{PMI}$ 
indicates in its application that IQOS mimics cigarette smoking better than e-cigarettes or vaping because of more rapid nicotine delivery, suggesting that IQOS may be much more effective at addicting youth and young adults to nicotine as well as increasing transitions to cigarette smoking. A net increase in nicotine addiction and cigarette uptake among adolescents and young adults is a realistic possibility that the PHIM does not consider.

Transition rates are one of the key parameters in the model, and their correct estimation is critical to the results.

\section{The model uses the 1990 US population and smoking prevalence as the starting point for the simulations}

The PHIM simulates the health impact on the population starting with a baseline population and smoking prevalence representative of the USA in 1990.(Module 6.5.2.2) ${ }^{12}$ It is not clear why 1990 data was used, when smoking prevalence was much greater than in more recent years; data for 2015 were readily available at the time of the analyses. Other published models use more recent prevalence data from 2000, ${ }^{8} 2006,{ }^{10} 2011^{6}$ and $2016 .{ }^{3}$ The use of 1990 prevalence is likely to lead to higher than actual smoking-attributable costs and higher expected benefits from IQOS.

\section{The PHIM ignores other tobacco products, such as e-cigarettes}

The population health results would be different if the PHIM comparison were between IQOS and a lower-risk product such as e-cigarettes. There are reasons to expect that e-cigarette users may find IQOS to be a tempting and attractive product, and ignoring the role of e-cigarette use in a model of the population health impact of IQOS will lead to an incomplete analysis.

The PHIM assumes very low rates of transition to dual use, contrary to empirical evidence from other countries showing that many of those individuals who use IQOS will continue to use their previous product. In Japan, where IQOS products are now available, over one-third of IQOS users are polyusers, most of whom also smoke cigarettes. ${ }^{25}$ Dual use of electronic tobacco products (HTP products including IQOS, Glo and Ploom Tech, or non-nicotine e-cigarettes) and combustible cigarettes was reported by 3.4\% of Japanese internet survey respondents in $2017 .{ }^{29}$ Thus, actual evidence of dual IQOS and cigarette use indicates that the assumptions of dual use rates in the PHIM are too low.

\section{Impact of IQOS on non-users is not considered}

Ignoring the impact on non-users who experience health risks from IQOS is not reasonable. Empirical evidence already exists for second-hand exposure from HTP aerosol. A Greek study found that nicotine levels for IQOS aerosol were greater than those in e-cigarettes at low puff duration, though lower than tobacco cigarettes. ${ }^{30}$ Another study using an animal model that exposed rats to cigarette smoke and IQOS aerosol at levels that were relevant to real-world human exposure levels found that both exposures resulted in similar vascular impairment. ${ }^{31}$ There is also direct evidence of negative health impacts from exposing human non-users to HTP aerosol. In Japan, 49\% of never-tobacco users and $41 \%$ of former tobacco users exposed to secondhand HTP aerosol reported symptoms including general illness, eye discomfort or a sore throat. ${ }^{29}$

Children are particularly likely to be impacted by exposure to HTP products. They may suffer negative health effects when exposed to their parents' second-hand aerosol, as they are when exposed to second-hand cigarette smoke. ${ }^{32-34}$ A Canadian study found that children suffered respiratory effects from exposure and digestive effects of ingestion of e-cigarettes. ${ }^{35}$ Women who use IQOS while pregnant may cause lifelong health impacts for their children, as is the case for women who smoke cigarettes or use snuff while pregnant. ${ }^{203637}$ Another potential risk from IQOS use is fires and explosions, such as those that occur with e-cigarettes. Ignoring the health impact of IQOS on non-users overestimates the benefit of IQOS as an MRTP. While this impact may be of a smaller magnitude than the impact on users of IQOS or cigarettes, the impact on non-users is recommended by the FDA for consideration.

\section{CONCLUSION}

The PHIM has a structure not unlike other simulation models reviewed. However, because it is used to justify the marketing of a tobacco product as a MRTP, it must satisfy FDA guidelines that other models are not subject to. The FDA is likely to receive a number of applications for MRTP orders in the coming years, and it is important that reasonable criteria be established for reviewing them. Future analyses of the impact of new tobacco products used for social decision-making such as regulatory actions should consider all relevant and substantial social effects. This includes both morbidity and mortality that arise from a comprehensive list of tobacco-attributable diseases. Model-based estimates need to carefully document methods for estimating key parameters such as transition rates and to validate model's predictive performance. Also, the effects of policy on all populations that will be affected should be included in the analyses, including non-tobacco users who will suffer health effects. These recommendations are relevant for the evaluation of new tobacco products as well as potential harm-reduction products more generally.

PMI, through its analysis of IQOS using the PHIM, has not shown that this product would 'significantly reduce harm and the risk of tobacco-related disease to individual tobacco users;

\section{What this paper adds}

- Heated tobacco products (HTP), also referred to as heat-notburn products, are not currently marketed in the USA and their impact on the health of the US population is not known.

- Philip Morris International developed a Population Health Impact Model that they used to estimate the potential impact of marketing an HTP, IQOS, as a modified-risk tobacco product (MRTP) in the USA.

- Because the model is used to support an MRTP application, the Food and Drug Administration guidelines indicate that it should include the impact of the new product on morbidity and mortality, and the impact on seven population groups and exposure patterns. However, the model underestimates mortality, omits morbidity measures, excludes impacts on non-users and underestimates the impact on other groups. Therefore, the model underestimates the potential impact of IQOS on the population as a whole and does not justify marketing the product as an MRTP.

- An assessment of the impact of a new tobacco product on population health should include a comprehensive measure of health impacts, consideration of all groups impacted, and documented and justifiable assumptions regarding model parameters such as the relative harm of the new product compared with existing products and transition rates between tobacco use categories. 
and benefit the health of the population as a whole taking into account both users of tobacco products and persons who do not currently use tobacco products'.(p3) ${ }^{14}$ As new tobacco products are introduced into US and worldwide markets, particularly those that purport to be less harmful than currently used products, models of population health impacts will play an important role. The PMI PHIM as applied to the marketing of IQOS as a MRTP illustrates some of the potential pitfalls of analysis that should be avoided.

Contributors WBM drafted the paper and $\mathrm{JL}$ took the lead on statistical review in major revisions. All authors contributed to the analyses and reviewed and edited the manuscript.

Funding This work was supported by the US National Cancer Institute at the National Institutes of Health $(\mathrm{NIH})$ and Food and Drug Administration Center for Tobacco Products (P50 CA180890).

Disclaimer The content is solely the responsibility of the authors and does not necessarily represent the official views of the NIH or the FDA.

\section{Competing interests None declared.}

\section{Patient consent Not requried.}

Provenance and peer review Not commissioned; externally peer reviewed.

Data sharing statement There are no unpublished data from this study.

Open access This is an open access article distributed in accordance with the Creative Commons Attribution Non Commercial (CC BY-NC 4.0) license, which permits others to distribute, remix, adapt, build upon this work non-commercially, and license their derivative works on different terms, provided the original work is properly cited, appropriate credit is given, any changes made indicated, and the use is non-commercial. See: http://creativecommons.org/licenses/by-nc/4.0/.

\section{REFERENCES}

1 Weitkunat R, Lee PN, Baker G, et al. A novel approach to assess the population health impact of introducing a modified risk tobacco product. Regul Toxicol Pharmacol 2015:72:87-93.

2 Kalkhoran S, Glantz SA. Modeling the health effects of expanding e-cigarette sales in the United States and United Kingdom: a monte carlo analysis. JAMA Intern Med 2015:175:1671-80.

3 Levy DT, Borland R, Lindblom EN, et al. Potential deaths averted in USA by replacing cigarettes with e-cigarettes. Tob Control 2018;27:18-25.

4 Levy DT, Borland R, Villanti AC, et al. The application of a decision-theoretic model to estimate the public health impact of vaporized nicotine product initiation in the United States. Nicotine Tob Res 2017;19:149-59.

5 Soneji SS, Sung HY, Primack BA, et al. Quantifying population-level health benefits and harms of e-cigarette use in the United States. PLoS One 2018;13:e0193328.

6 Warner KE, Mendez D. E-cigarettes: comparing the possible risks of increasing smoking initiation with the potential benefits of increasing smoking cessation. Nicot Tob Res 2018.

7 Bachand AM, Sulsky SI, Curtin GM. Assessing the likelihood and magnitude of a population health benefit following the market introduction of a modified-risk tobacco product: enhancements to the Dynamic Population Modeler, DPM(+1). Risk Anal 2018;38:151-62.

8 Vugrin ED, Rostron BL, Verzi SJ, et al. Modeling the potential effects of new tobacco products and policies: a dynamic population model for multiple product use and harm. PLoS One 2015;10:1-26

9 Poland B, Teischinger F. Population modeling of modified risk tobacco products accounting for smoking reduction and gradual transitions of relative risk. Nicot Tob Res 2017:19:1277-83.

10 Mejia AB, Ling PM, Glantz SA. Quantifying the effects of promoting smokeless tobacco as a harm reduction strategy in the USA. Tob Control 2010;19:297-305.

11 Lee PN, Fry JS, Hamling JF, et al. Estimating the effect of differing assumptions on the population health impact of introducing a reduced risk tobacco product in the USA. Regul Toxicol Pharmacol 2017;88:192-213.

12 Philip Morris Products S.A. Modified Risk Tobacco Product (MRTP) Applications, Module 6: summaries of all research findings. https://www.fda.gov/TobaccoProducts/ Labeling/MarketingandAdvertising/ucm546281.htm-6

13 Philip Morris Products S.A. Modified Risk Tobacco Product (MRTP) Applications, Module 7: scientific studies and analyses. executive summary. https://www.fda.gov/ TobaccoProducts/Labeling/MarketingandAdvertising/ucm546281.htm-7
14 U.S. Department of Health and Human Services, Food and Drug Administration, Center for Tobacco Products. Guidance for industry. modified risk tobacco product applications. draft Guidance, 2012.

15 Goodchild M, Nargis N, Tursan d'Espaignet E. Global economic cost of smokingattributable diseases. Tob Control 2018;27:58-64.

16 Glantz SA. PMI's own in vivo clinical data on biomarkers of potential harm in Americans show that IQOS is not detectably different from conventional cigarettes. Tob Control 2018;27(Suppl1):s9-s12.

17 US Department of Health \& Human Services. The Health Consequences of Smoking -- 50 Years of Progress. A report of the surgeon general. Atlanta, GA: Department of Health and Human Services, Centers for Disease Control and Prevention, National Center for Chronic Disease Prevention and Health Promotion, Office on Smoking and Health, 2014.

18 Yao T, Max W, Sung H-Y, et al. Relationship between spending on electronic cigarettes, 30-day use, and disease symptoms among current adult cigarette smokers in the U.S. PLoS One 2017;12:e0187399.

19 Centers for Disease Control and Prevention. Atlanta, GAAdult SAMMEC: smokingattributable mortality, morbidity, and economic costs: Relative risk: Centers for Disease Control and Prevention, 2014.

20 Centers for Disease Control and Prevention. Maternal and child health (MCH) SAMMEC: smoking-attributable mortality, morbidity, and economic costs: relative risk Atlanta, GA: Centers for Disease Control and Prevention, 2014.

21 Liu X, Lugo A, Spizzichino L, et al. Heat-not-burn tobacco products: concerns from the Italian experience. Tob Control 2018:tobaccocontrol-2017-054054 [Epub ahead of print 26 Jan 2018].

22 U.S. Department of Health and Human Services. E-Cigarette use among youth and young adults: a report of the surgeon general. Atlanta, CA: US Department of Health and Human Services, Centers for Disease Control and Prevention, National Center for Chronic Disease Prevention and Health Promotion, Office on Smoking and Health, 2016.

23 Halpern-Felsher B, McKelvey K, Popova L, 2017. The evidence cited in PMI's MRTP Application indicates that the proposed labeling and warnings for IQOS will mislead consumers, particularly youth, about the product. Comment submitted to Docket Number: FDA-2017-D-3001. https://tobacco.ucsf.edu/evidence-pmi\%E2\%80\% 99s-mrtp-application-indicates-proposed-labeling-and-warnings-iqos-will-misleadconsumers

24 Pepper JK, Coats EM, Nonnemaker JM, et al. How do adolescents get their e-cigarettes and other electronic vaping devices? Am J Health Promot 2018;1:1-10.

25 van der Plas LP, Skiada D, Dobrynina M, et al, 2017. Prevalence and patterns of tobacco use in Japan after the commercialization of a heat-not-burn alternative (IQOS) to cigarettes. wwwpmisciencecom

26 Weaver SR, Huang J, Pechacek TF, et al. Are electronic nicotine delivery systems helping cigarette smokers quit? Evidence from a prospective cohort study of U.S. adult smokers, 2015-2016. PLoS One 2018;13:e0198047.

27 Selya AS, Dierker L, Rose JS, et al. The role of nicotine dependence in e-cigarettes' potential for smoking reduction. Nicotine Tob Res 2017.

28 Soneji S, Barrington-Trimis JL, Wills TA, et al. Association between initial use of e-cigarettes and subsequent cigarette smoking among adolescents and young adults: a systematic review and meta-analysis. JAMA Pediatr 2017;171:788-97.

29 Tabuchi T, Gallus S, Shinozaki T, et al. Heat-not-burn tobacco product use in Japan: its prevalence, predictors and perceived symptoms from exposure to secondhand heatnot-burn tobacco aerosol. Tob Control 2018;27(e1):e25-e33.

30 Farsalinos KE, Yannovits N, Sarri T, et al. Nicotine delivery to the aerosol of a heat-notburn tobacco product: comparison with a tobacco cigarette and E-Cigarettes. Nicotine Tob Res 2018:20:1004-9.

31 Nabavizadeh P, Liu J, Havel C, et al. Inhalation of heat-not-burn tobacco aerosol impairs vascular endothelial function. Tob Control 2018.

32 Mannino DM, Moorman JE, Kingsley B, et al. Health effects related to environmental tobacco smoke exposure in children in the United States: data from the Third National Health and Nutrition Examination Survey. Arch Pediatr Adolesc Med 2001;155:36-41.

33 Stoddard JJ, Gray B. Maternal smoking and medical expenditures for childhood respiratory illness. Am J Public Health 1997;87:205-9.

34 Max W, Sung H-Y, Shi Y. Attention deficit hyperactivity disorder among children exposed to secondhand smoke: a logistic regression analysis of secondary data. Int J Nurs Stud 2013:50:797-806.

35 Richmond SA, Pike I, Maguire JL, et al. E-cigarettes: a new hazard for children and adolescents. Paediatr Child Health 2018;23:255-9.

36 Gunnerbeck A, Wikstrom A-K, Bonamy A-KE, et al. Relationship of maternal snuff use and cigarette smoking with neonatal apnea. Pediatrics 2011;128:503-9.

37 Langley $\mathrm{K}$, Rice $\mathrm{F}$, van den Bree MB, et al. Maternal smoking during pregnancy as an environmental risk factor for attention deficit hyperactivity disorder behaviour. A review. Minerva Pediatr 2005;57:359-71. 